\title{
Prospective evaluation of the diagnostic accuracy of liver ultrasonography
}

\author{
J C DEBONGNIE,* C PAUls, M FIEVEZ, AND E WIBIN
}

From the Departments of Internal Medicine, Radiology, and Surgery, Clinique St-Pierre, 1340 Ottignies, Belgium, and the Institut de Morphologie Pathologique, Loverval, Belgium

SUMMARY Liver ultrasound was prospectively evaluated in 104 subjects who underwent liver biopsy, including 24 patients without evidence of liver disease (controls), and 80 with a broad spectrum of liver pathology. Ultrasonography was very specific $(100 \%)$ and moderately sensitive $(70 \%)$ in the detection of liver pathology, and hepatic neoplasms, steatosis, and fibrosis were detected by ultrasound in $80 \%, 80 \%$, and $67 \%$ of cases respectively. In addition, ultrasonography diagnosed other pathologies-mainly biliary tract disease and abdominal neoplasms-in $26 \%$ of the patients.

Ultrasound is an 'effective regional-imaging device based on morphology'. ${ }^{1}$ Gray-scale signal processing allows a detailed analysis of texture patterns of solid organs and thus the characterisation of both diffuse pathological processes and space-occupying lesions. The contours and the echotexture of internal organs are outlined and changes in adjacent viscera delineated. Real-time imaging decreases examination time, and allows a quick and complete survey of the region of interest.

Liver ultrasound thus has the ability to detect diffuse as well as focal liver disease by analysis of the liver texture and may define associated abnormalities such as ascites or splenomegaly. Different patterns of metastatic liver disease have been described ${ }^{2}{ }^{3}$ and an accuracy of $90 \%$ has been claimed. ${ }^{4}$ Diffuse hepatocellular disease may produce changes in liver echo pattern and an accuracy of $81 \%$ has been recorded in a retrospective study. ${ }^{5}$

In order to evaluate the accuracy, sensitivity, and specificity of liver ultrasound, a prospective study was undertaken of patients undergoing liver biopsy for evaluation of liver disease and controls undergoing abdominal surgery for various reasons.

\section{Methods}

PATIENTS

A careful ultrasonographic examination of the liver was performed in 110 consecutive patients before

*Address for correspondence and reprint requests: Dr J C Debongnie, Département de Médecine Interne, Clinique St-Pierre, B-1340 Ottignies, Belgium.

Received for publication 5 September 1980. liver biopsy between November 1978 and October 1979. All patients with obstructive jaundice or previously diagnosed liver disease were excluded.

Six patients were excluded: in four patients the liver biopsy was inadequate, including less than three portal spaces; and in two patients with probable liver tumour a final diagnosis was not reached as surgical biopsy was not performed after a negative peritoneoscopy.

In 85 patients, the liver biopsy was performed because a liver problem was suspected. Five had a normal liver on biopsy. In 22 patients with a presumed normal liver, including 12 patients with an intra-abdominal neoplasm and seven with benign conditions, all requiring surgery, a liver biopsy was obtained during laparotomy. All gave informed consent concerning the liver biopsy. Three of those 22 patients had an abnormal liver on biopsy (two neoplasms, one cirrhosis).

All patients undergoing liver ultrasonography and liver biopsy had a full clinical examination by the same examiner (JCD) and the following blood tests: haemogram, transaminases, alkaline phosphatase, gamma-glutamyl transferase, bilirubin, prothrombin time, serum electrophoresis. Nine patients had ascites, eight a clinically enlarged spleen, and 12 jaundice. Serum albumin was less than $3 \mathrm{~g} / \mathrm{l}$ in seven patients, prothrombin time was below $50 \%$ in two.

\section{LIVER ULTRASONOGRAPHY}

Sonograms were obtained using two commercially available gray scale ultrasonoscopes.

A rapid real time abdominal survey (Toshiba Sonolayer-graph real-time unit using an electronic- 

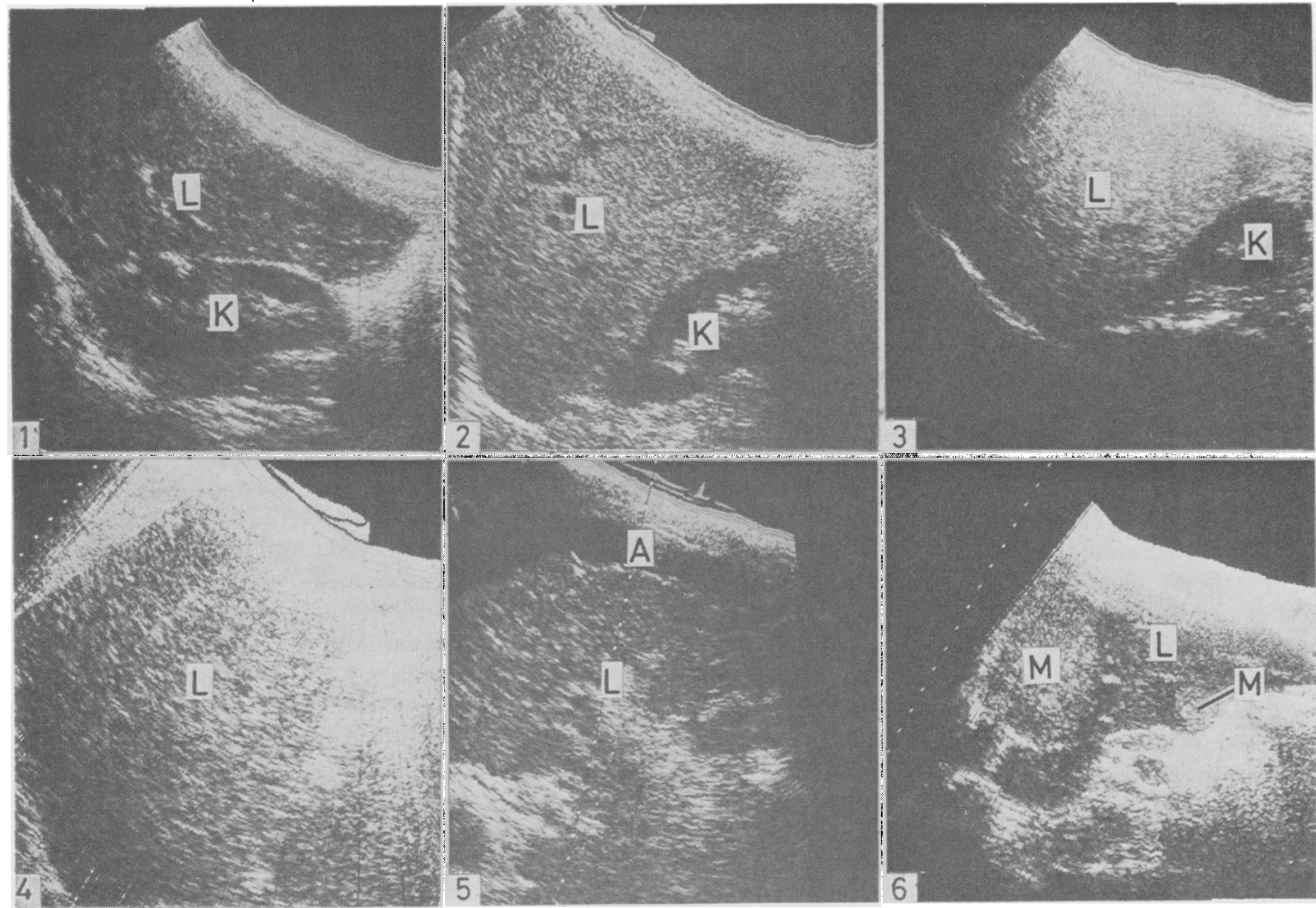

Fig. 1 Normal liver. Longitudinal section through the right lobe of the liver and the kidney. L:liver. K:kidney. Fig. 2 Steatosis. Section similar to Fig. 1. Increased echogenicity as compared with the renal parenchyma with small and tightly packed echo targets. Normal beam penetration. Fig. 3 Steatofibrosis. Section similar to Fig. 1. Increased echogenicity with decreased beam penetration.

Fig. 4 Alcoholic (micronodular) cirrhosis. Section similar to Fig. 1. Marked coarse bright pattern with distal attenuation of the sound beam. Fig. 5 Posthepatitic (macronodular) cirrhosis. Oblique subcostal scan through the right lobe. Coarse echo targets. Normal overall echogenicity. Normal beam penetration. A:ascites. Fig. 6 Metastatic liver. Longitudinal midline section. Two hyperechogenic metastases measuring respectively 6 and $2 \mathrm{~cm}$. M:metastases.

ally driven 64-element linear-array transducer2,4 and 3,5 MHZ) was first performed, allowing examination of the pleural and peritoneal spaces for effusion and other pathological conditions and detailed imaging of all portions of the hepatic parenchyma.

A more detailed analysis of the hepatic parenchyma was obtained by standard laminagraphic Bscan sections (Searle Pho/Sonic SM gray scale unit with a 2,25 MHZ, $19 \mathrm{~mm}$ long focus transducer). Manual B scanning was particularly useful for evaluation of diffuse hepatocelluler disease, allowing examination of a greater anatomical field, including a large volume of the right liver lobe, the diaphragm, and the kidney, a better control of the different instrument settings and A-scan analysis (not available on the real time unit). The diagnostic echo patterns were recognised at standard instrument settings, although higher gain settings were frequently required for optimal results in obese patients.

Representative scans are shown in Figs. 1 to 6. The association of hyperechogenic liver tissue (as compared with the kidney) with fine and tightly packed echo targets, normal beam penetration, and decrease in vascularity was characteristic of steatosis (Fig. 2). Associated decreased beam penetration suggested the additional presence of fibrosis (Fig. 3). Coarse echo targets indicated the presence of fibrosis, and, when marked, the presence of cirrhosis especially if associated with hepatic marginal irregularities, ascites, splenomegaly, or an enlarged portal vein (Figs. 4 and 5). Different previously published ${ }^{0}$ ultrasonic patterns were considered as 
indicative of tumour: hyperechogenic, hypoechogenic or necrotic masses, diffusely heterogeneous pattern (Fig. 6).

The information was immediately recorded on a standard sheet and the conclusions stated as follows: normal or abnormal examination; focal or diffuse abnormality; specific diagnosis (tumour, cirrhosis, etc.). The mean examination time measured on 10 patients was 10 minutes. All sonograms were performed by the same examiner (CP).

\section{LIVER BIOPSY}

Percutaneous liver biopsy was obtained in 48 patients using a modified Menghini needle (Jamshidi $70 \times$ $1.4 \mathrm{~mm}$ or $80 \times 1.8 \mathrm{~mm}$ ). Liver biopsy was performed during peritoneoscopy in 24 patients using a Menghini needle $(1.6 \mathrm{~mm})$. A surgical wedge liver biopsy was obtained in 34 patients. The diagnosis was reached in four patients by cytological examination after needle aspiration (three amoebic abcesses, one cystic necrotic metastatis).

All specimens were reviewed by the same pathologist (MF). Diagnostic interpretation followed standard criteria. No attempt was made to quantify necrosis, fatty infiltration, or fibrosis. The main diagnostic feature was recorded as the final diagnosis -for instance, alcoholic hepatitis associated with steatosis and fibrosis was recorded as alcoholic hepatitis.

\section{Result}

\section{HISTOLOGICAL DIAGNOSIS}

In the 24 control patients, liver histology was normal. In the 80 patients with liver disease tumour was diagnosed in 20 , cirrhosis in 20 , and miscellaneous lesions in $\mathbf{4 0}$ (hepatitis, nine; vascular lesions, four; steatosis and/or fibrosis, 22; focal benign lesions, four; drug-induced intrahepatic cholestasis, one).

OVERALL RESULTS: ULTRASONIC DETECTION OF LIVER DISEASE

All control patients had a normal ultrasonogram (Table 1). Fifty-six of the 80 patients with abnormal liver tissue were diagnosed as abnormal by ultra-

Table 1 Ultrasonic detection of liver disease

\begin{tabular}{llcr}
\hline Tissue & \multicolumn{2}{l}{ Ultrasound diagnosis } \\
\cline { 2 - 4 } & Normal & Abnormal & \\
\hline Normal & 24 & 0 & 24 \\
Abnormal & 24 & 56 & 80 \\
Totals & 48 & 56 & 104 \\
\hline
\end{tabular}

Specificity: $100 \%$. False positive: $0 \%$.

Sensitivity: $70 \%$. False negative: $30 \%$. sonographic criteria. Half of the false negative patients on ultrasound had mild histological changes on biopsy.

Thus, in this series, ultrasound is very specific $(100 \%)$, as no false positive was obtained, and moderately sensitive, as $70 \%$ of the abnormal group were correctly diagnosed.

\section{Tumour}

Liver tumour was primary in two patients, secondary in 18 patients; lesions were solitary in five patients (with a size between 3 and $10 \mathrm{~cm}$ ), restricted to one lobe in two patients, and diffuse in 13 patients.

Ultrasound diagnosed liver tumour in 16 patients, diffuse liver disease in two patients. In two cases, the liver was considered normal by both the ultrasonographer and the surgeon at laparotomy. However, microscopic examination of liver tissue from these two cases disclosed diffuse neoplastic involvement with carcinoid tumour in one patient and a subcapsular neoplastic deposit in the second.

Various ultrasonic patterns were observed: hyperechogenic (five), hypoechogenic (four) or necrotic masses (two), diffusely heterogeneous pattern (five).

\section{Cirrhosis}

The aetiology of cirrhosis was alcohol in 12 patients, hepatitis in six (including two with chronic active hepatitis), haemochromatosis in one, and unknown in one.

Ultrasonography correctly diagnosed liver cirrhosis in 11. Diffuse liver disease (fibrosis and/or steatosis) was diagnosed in six patients. The liver was considered normal in three patients with macronodular cirrhosis including two with chronic active hepatitis. Ultrasound correctly suggested the presence of fat in 11 of the 12 alcoholic cirrhotics.

\section{Hepatitis}

Ultrasound revealed no abnormality in the patients with acute hepatitis (three), chronic persistent hepatitis (one), chronic active hepatitis without cirrhosis (one), lobular hepatitis (one), but steatosis and fibrosis was detected in three patients with alcoholic hepatitis.

\section{Fat-fibrosis}

This group includes 14 patients with steatosis (three with severe steatosis), two with fibrosis and six with steatosis and fibrosis. Ultrasound findings were abnormal in 15 of the 22 patients. Five patients with minimal or moderate steatosis and two patients with fibrosis were considered normal.

\section{Miscellaneous}

The liver was considered normal by the ultrasonographer in the four patients with 'vascular' lesions 
(central vein dilatation, centrolobular necrosis), and pericardial effusion and dilatation of the hepatic veins and inferior vena cava were observed by ultrasound in one patient. Three patients with an amoebic abscess and one patient with a liver cyst were correctly diagnosed by ultrasound. In one patient with a drug-induced cholestatic jaundice, the liver was considered normal.

\section{ACCURACY OF ULTRASONIC TISSUE}

\section{DIAGNOSIS}

The presence of liver cell necrosis, tumour, steatosis, and fibrosis was recorded separately for each patient and its detection rate by ultrasound is summarised in Table 2. For example, a final diagnosis of alcoholic hepatitis may include liver cell necrosis, steatosis, and fibrosis. When present, tumour was diagnosed by ultrasound in $80 \%$ of the patients, fat in $80 \%$, fibrosis (including cirrhosis) in 67\%, and liver cell necrosis in $0 \%$. In two patients with pure steatosis, ultrasound suggested additional fibrosis. In one cirrhotic patient, ultrasound falsely diagnosed steatosis. In those three patients, ultrasound was thus abnormal but gave an incorrect interpretation.

\section{INTRAOBSERVER VARIATION OF READING}

Photographic records of the ultrasound study were read blindly after completion of the study by the same radiologist and reported as normal or abnormal. Satisfactory records were available for 77 cases. All controls $(n=16)$ were classified as normal and 52 of the patients with liver disease $(n=61)$ were considered as abnormal. At the first reading, 49 of the same patients were considered abnormal. In seven patients, a discrepancy was observed between the

Table 2 Ultrasonic tissue diagnosis

\begin{tabular}{lll}
\hline Tissue diagnosis & No. & Accurate ultrasound diagnosis \\
\hline Tumour & 20 & $16(0)$ \\
Steatosis & 35 & $28(1)^{*}$ \\
Fibrosis & 31 & $21(2)^{*}$ \\
Liver cell necrosis & 11 & $0(0)$ \\
\hline
\end{tabular}

- In these patients, steatosis (1) and fibrosis (2) were suggested on ultrasound but absent on biopsy.

Table 3 Ultrasonic associated findings

\begin{tabular}{|c|c|c|}
\hline Finding & No. & Diagnosis \\
\hline Gallbladder & 15 & Lithiasis \\
\hline Bile ducts & 1 & Choledochal cyst \\
\hline \multirow[t]{3}{*}{ Neoplasm } & 6 & Gastric (2) \\
\hline & & Small bowell (1) \\
\hline & & $\begin{array}{l}\text { Lymphoma (1) } \\
\text { Pancreas (2) }\end{array}$ \\
\hline Miscellaneous & 5 & $\begin{array}{l}\text { Chronic pancreatitis (2) } \\
\text { Pericardial effusion (2) }\end{array}$ \\
\hline & & Dilated hepatic veins (1) \\
\hline Total & 27 & \\
\hline
\end{tabular}

two readings; two of the patients had cirrhosis, three steatosis, and two steatosis and fibrosis.

Thus both readings gave similar overall results and intraobserver variation of the readings was $9 \%$.

\section{ASSOCIATED FINDINGS}

During the regional ultrasonic survey, extrahepatic pathology was detected in 27 patients $(26 \%)$. Those findings were unknown before the ultrasound study in 19 patients and included mainly biliary (16) and neoplastic (six) disease (Table 3).

\section{LIVER SCINTIGRAPHY}

Standard isotopic examination of the liver after injection of $69 \mathrm{Tc}$ sulphur colloid was performed in 72 cases -10 controls and 62 patients with liver disease Two of the controls had an abnormal test, and 15 of the patients had a normal test. The test was normal in two patients with liver tumour $(n=17)$. In four patients with extrahepatic tumour focal defects were suggested by the test but disproven by ultrasound and at surgery or peritoneoscopy.

\section{Discussion}

In assessing the efficacy of a diagnostic test, interpretation of the results and establishment of the actual diagnosis should be carried out independently and a broad spectrum of pathology and 'healthy' controls examined. ${ }^{6}$ These conditions were fulfilled in the present study. The ultrasonic diagnosis was obtained before liver biopsy in all patients. In the 24 controls, liver biopsy was normal and visualisation of the liver at surgery or peritoneoscopy in 21 subjects revealed no abnormality. Among the 80 patients, less than $10 \%$ had signs of severe liver disease such as ascites, a low serum albumin, or a prothrombim time less than $50 \%$. In the 36 alcoholic patients (defined as consuming more than $80 \mathrm{~g}$ of ethanol/day for more than five years), the final diagnosis was: normal liver in four, steatosis in nine, steatosis and/or fibrosis in eight, hepatitis in three, cirrhosis in 12. We therefore consider that we have included adequate controls and a wide spectrum of liver pathology to enable evaluation of ultrasound as a diagnostic tool in liver disease.

All controls had a normal ultrasonogram. Thus, the specificity was $100 \%$, as no control had an abnormal test (no false positive), and the predictive value of a positive test was $100 \%$, as all patients with an abnormal ultrasonogram had liver disease. In two retrospective evaluations of ultrasonography in hepatocellular disease, a false positive rate of respectively $0 \%$ and $24 \%$ was obtained. ${ }^{5} 7$ If ultrasonography is to be considered as a screening test for 
liver disease, a high specificity is very important. ${ }^{8}$ In an unselected population of 10000 subjects, with an arbitrarily chosen prevalence of liver disease of $2 \%$, the positive predictive value of the test with a sensitivity of $70 \%$ would be $100 \%$ if the test has no false positive, but only $0.06 \%$ if it has $24 \%$ false positive. The specificity of liver ultrasound should thus be confirmed in a large series.

In this series, $70 \%$ of patients with an abnormal liver on biopsy had an abnormal ultrasonogram. The test is thus moderately sensitive but this varies according to the liver pathology.

In 20 patients with liver tumour, the ultrasound study was abnormal in $90 \%$, correctly diagnosing cancer in $80 \%$. In retrospective studies, the accuracy of ultrasound varies between $75 \%$ and $90 \%{ }^{11-14}$

In 20 patients with liver cirrhosis, the ultrasonogram was abnormal in $85 \%$, correctly diagnosing cirrhosis in $55 \%$. In a recent retrospective study a correct diagnosis was obtained in $57 \%$ of ultrasonic scans, the test being abnormal in $81 \% \%^{6}$ In another study, a 'bright liver echo pattern' was found in $65 \%$ of patients, a normal pattern in $35 \%$, and ultrasound detected $80 \%$ of micronodular cirrhosis, while missing $80 \%$ of macronodular cirrhosis. ${ }^{7}$ In our series, ultrasound was normal in three of the six patients with macronodular cirrhosis.

In this series, the technique identified three quarters of patients with steatosis and/or fibrosis. The presence of fat is easily recognised by ultrasound, ${ }^{10}$ although its presence in the liver is nonspecific and may be observed in a variety of conditions such as alcoholism, diabetes, drugs, porphyria. When ultrasound diagnosed cirrhosis associated with steatosis, all patients in the present series had alcoholic liver disease, although this association may be caused by rarer conditions such as Wilson's disease. All patients with hepatitis unassociated with steatosis had a normal ultrasonogram. In summary, ultrasound is capable of detecting replacement of normal tissue by fat, fibrosis, and tumour in a significant proportion of patients and is therefore a useful diagnostic aid in patients with suspected alcoholic liver disease or hepatic neoplasm.

The value of ultrasound as a regional-imaging device is supported by the additional extrahepatic pathology detected in $26 \%$ of the patients. Such findings may help clarify the nature of abnormalities observed in the liver scans, such as dilated ducts or a space-occupying lesion. It may exclude obstruction of the biliary tract or detect cholelithiasis in patients with chronic liver disease, both of which are difficult to demonstrate by standard contrast radiology. These advantages of ultrasonography over liver scintigraphy would suggest that it is suitable as a 'first line' diagnostic procedure.
This study does not allow a true comparison with liver scintigraphy, as only $70 \%$ of the patients had this test, including less than half of the controls and most of the patients with more severe liver disease. As most of the false positives of liver scintigraphy are caused by anatomical variations, ultrasound has been recommended as a complementary test in such cases, ${ }^{11}{ }^{12}$ as well as in focal disease for elucidating the liquid or solid nature of a lesion. ${ }^{13}$

The major disadvantage of liver ultrasound is its dependance on the radiologist. The description of diffuse changes is mainly qualitative and only selected records of the examination are available. Thus, this test does not fulfil one of the requirements detailed in a recent workshop on diagnostic techniques in hepatobiliary disease $:^{14}$ the definition of a predetermined diagnostic cut-off point for the presence or absence of a lesion. However, intraobserver variation of the readings was minimal in this study; interobserver variation was $18 \%$ in another study. ${ }^{5}$ Therefore, despite qualitative and somewhat subjective results, interpretation of the test is reproducible. Current progress is being made towards a more quantitative approach by ultrasonic diffraction analysis. ${ }^{15}$

\section{References}

${ }^{1}$ Ferruci JT. Body ultrasonography. $N$ Engl J Med 1979; 300: 538-45, 590-602.

${ }^{2}$ Green B, Bree RL, Goldstein HM, Stanley C. Gray scale ultrasound evaluation of hepatic neoplasms: patterns and correlations. Radiology 1977; 124: 203-8.

${ }^{3}$ Scheible W, Gosink BB, Leopold GR. Gray scale echographic patterns of hepatic metastatic disease. AJR 1977; 129: 983-7.

${ }^{4}$ Taylor KJW, Carpenter DA, Hill CR, McCready VR. Gray scale ultrasound imaging. The anatomy and pathology of the liver. Radiology 1976; 119: 415-23.

${ }^{5}$ Gosink BG, Lemon SK, Scheible W, Leopold GR. Accuracy of ultrasonography in diagnosis of hepatocellular disease. $A J R$ 1979; 133: 19-23.

${ }^{6}$ Ransohoff DF, Feinstein AR. Problems of spectrum and bias in evaluating the efficacy of diagnostic tests. $N$ Engl J Med 1978; 299: 926-30.

${ }^{2}$ Dewburry KC, Clark B. The accuracy of ultrasound in the detection of cirrhosis of the liver. Br J Radiol 1979; 52: $945-8$.

${ }^{8}$ Vecchio ThJ. Predictive value of a single diagnostic test in unselected populations. $N$ Engl J Med 1966; 274: 1171-3.

${ }^{9}$ Snow JG, Goldstein HM, Wallace S. Comparison of scintigraphy, sonography and computed tomography in the evaluation of hepatic neoplasms. AJR 1979; 132: 915-8.

${ }^{10}$ Behan M, Kazam E. The echographic characteristics of fatty tissues and tumours. Radiology 1978; 129: 143-51. 
"Sullivan DC, Taylor KJW, Gottschalk A. The use of ultrasound to enhance the diagnostic utility of the equivocal liver scintigraph. Radiology 1978; 128: 727-32

22Taylor KJW, Sullivan D, Rosenfield AT, Gottschalk A. Gray scale ultrasound and isotope scanning: complementary techniques for imaging the liver. AJR 1977; 128: 277-81.

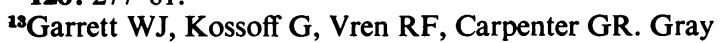

scale ultrasonic investigation of focal defects on $99 \mathrm{mTc}$ sulphur colloid liver scanning. Radiology 1976; 119: 425-8.

${ }^{14}$ Conn HO. AGA Workshop-Diagnostic techniques in hepatobiliary disease. Gastroenterology 1978; 75: 1175-7.

${ }^{15}$ Nicolas D. Ultrasonic diffraction analysis in the investigation of liver disease. Br J Radiol 1979; 52: 949-61. 